\title{
A Hospital Medical Gas Monitoring System Based on Multi-mode Optical Fiber Ring Network
}

\author{
Wei Sun ${ }^{1}$, Jian Wang ${ }^{1}$, Li-Hong Gai ${ }^{2}$, Jun-Jie Gu${ }^{2}$ and Li Li ${ }^{1}$ \\ ${ }^{1}$ School of Mechatronic Engineering and Automation, Shanghai University, 200072 Shanghai, China \\ ${ }^{2}$ Environmental Engineering CO, LTD of Shandong Academy of Environmental Science, 250013 Jinan, China
}

\begin{abstract}
This paper proposes a hospital medical gas monitoring system based on optical fiber ring network aiming to solve the problems of current medical gas monitoring, for example, the function of acquisition points is single, data transmission is not real-time, and communication distance is short. This system can display real-time gas state data and alarm in time by using the multi-function regional monitoring alarms as monitoring nodes. Meanwhile, we use multi-mode optical fiber ring network to connect the monitoring nodes throughout the hospital because of a longdistance transmission. Experimental results show that the system has the features of multifunction, safety, reliability, convenience and utility.
\end{abstract}

\section{Introduction}

Medical gas project is known as one of the life support system, whose normalization, safety, reliability and reasonability directly related to the patient's life safety [1]. The function of medical gas monitoring system is providing real-time state data of medical gas system to device managers or medical staffs. Once the parameters are out of the normal range, it emits sound and light alarm signal. Most of the existing hospital medical gas monitoring systems use the method of installing the pressure or flow meters in the gas equipment as gas parameter acquisition node. This gas parameter acquisition node has only a single function, so it need artificial screening. When gas failure occurs, alarm occurs only in control table, which is unfavorable for medical staff to notice the gas breakdown in first time.

Currently, monitoring center and regional acquisition nodes of existing hospital medical monitoring systems communicate with each other through fieldbus. However, in the field environment, the effective transmission distance (such as RS485, CAN, etc.) of common fieldbus is generally only 300 or 400 meters, and their transmission rate is $100 \mathrm{kbit} / \mathrm{s}$ or less. In addition, since most of major hospitals cover large area so that the gas region disperses in different locations of the hospital, which result in that most gas regions are far from the monitoring center, so the distance exceeds common fieldbus's effective transmission distance. Therefore, connecting monitoring center and gas religions with fieldbus cannot meet all the requirements in terms of transmission distance, transmission speed or data bandwidth. Compared with the fieldbus, multi-mode fiber supports longer transmission distance, it can support up to 2000 meters' transmission distance when used in $10 \mathrm{Mbps}$ and $100 \mathrm{Mbps}$ Ethernet. In this system, gas state data acquisition nodes of each regional monitoring subnet communicate with each other through fieldbus, regional monitoring subnets throughout the hospital are connected by multi-mode fiber ring network [2].

\section{Overall system design scheme}

The overall design scheme of the system shown in Fig. 1. The monitoring center is composed by the industrial personal computer (IPC), the printer and the switch. The switch is responsible for transforming the optical signals of multi-mode optical fiber ring network into electrical signals for IPC, at the same time, it transforms the electrical signal emitted by IPC into optical signals which can transmit in a multi-mode optical fiber ring network. Each gas region form a set of regional monitoring subnets in building, each floor place one or two regional monitoring alarm as regional monitoring nodes. Microprocessor is embedded in regional monitoring alarm, who not only can send the data measured by the sensors to the fieldbus, but also has function of real-time display and alarm.

The PLC with communication interface modules collects and processes data from fieldbus, then control the human-machine interface which we called regional monitoring panel to display the gas state data acquired from every acquisition node, RTU/TCP gateway achieve the function of mutual conversion between Modbus RTU and Modbus TCP protocols [3]. The medical gas supply stations such as negative pressure pump station, air pressure pump station and liquid oxygen station equipped with the same devices. PLC and monitoring center 
communicate via switches and multimode optical fiber ring network.

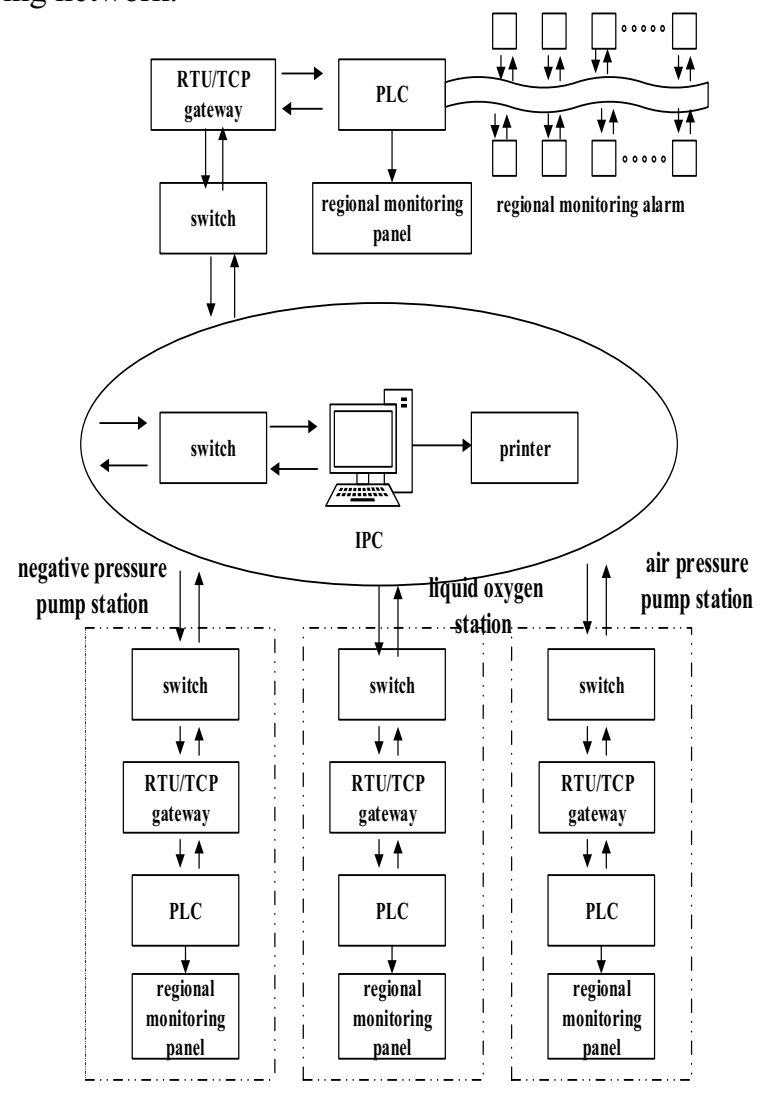

Figure 1. Overall system design scheme.

The IPC in monitoring center can monitor the hospital medical gas state of overall hospital, it has a function of historical data query, and the gas state data can be printed when needed. Regional monitoring panel can also set the upper and lower limit alarm values manually. Fieldbus and multi-mode optical fiber ring network two means of communication methods are used in this system in order to take full advantage of their advantages. In the process of monitoring, if the multi-mode fiber optic ring network breakage occurs somewhere for any reason, optical fiber ring network break into ordinary optical fiber line, while it is still capable of transmit optical signals which can ensure the normal communication [4].

\section{Important modules of system}

The hospital medical gas monitoring system based on multi-mode optical fiber ring network includes several important modules to ensure achieve the desired results, such as regional monitoring alarm and regional monitoring panel.

\subsection{Formatting the title, authors and affiliations}

Regional monitoring alarm is composed by modular splicing, each function module can display or monitor two kinds of gas data simultaneously. If you need to monitor multiple gases simultaneously, more function modules can be spliced together [5]. The hardware structure of each function module is shown in Fig. 2. It includes the modules of microprocessor (MCU), power supply circuit, clock circuit, input signal selection circuit, display driver, digital display, keys, DIP switch, buzzer, data storage circuit and fieldbus communication circuit.

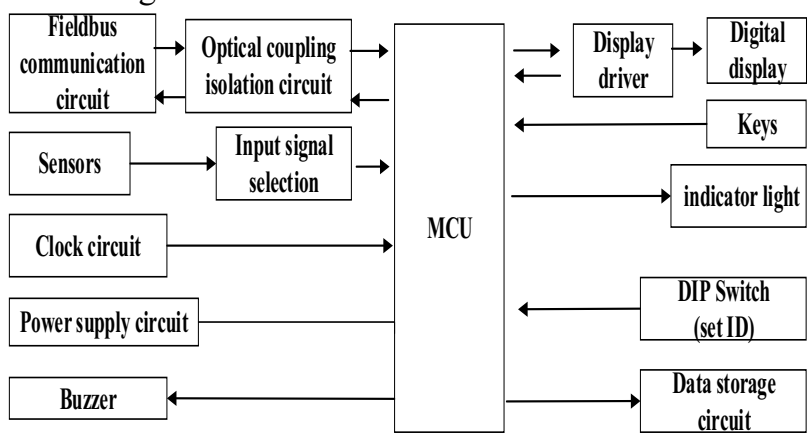

Figure 2. Hardware architecture of regional monitoring alarm.

Main software workflow of regional monitoring alarm is shown in Fig. 3, the MCU's analog channels receive the value of medical gas pressure, and its analog / digital (A / D) converter convert analog value, the converted data is stored after computed and processed by MCU, the display module calls the data and displays real-timely [6]. At the same time, communication module send data to the fieldbus. MCU compare the medical gas data with threshold value. If the data is higher than the upper limit, light on the indicator light "high", the buzzer sound the alarm; similarly, if the data is lower than the lower limit, light on the indicator light "low", the buzzer also sound the alarm. If the mute button is pressed when the buzzer is ring, the alarm sound pause for some time.

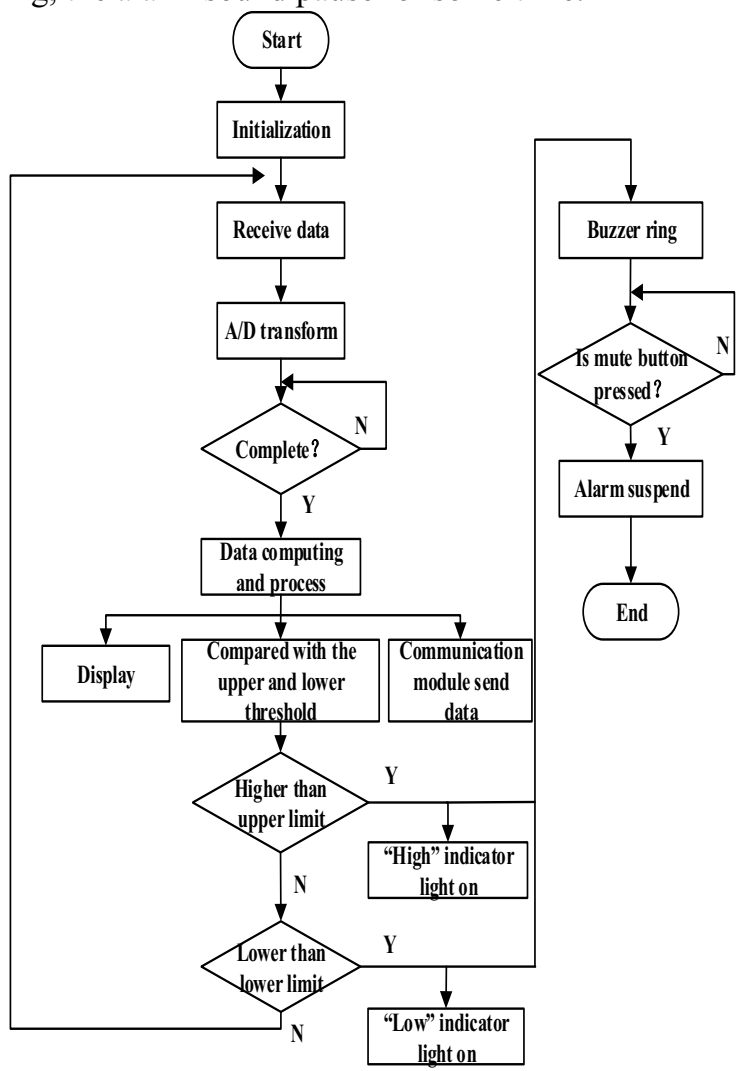

Figure 3. Hardware architecture of regional monitoring alarm. 


\subsection{Regional monitoring panel}

The function of regional monitoring panel is monitoring all acquisition nodes of one of the buildings or medical gas supply station. If medical gas fault occurred in somewhere, regional monitoring panel sound the alarm, and what's more, it can show the fault position [7]. By operating directly on the touch screen, you can set the threshold value of the medical gas state data, and you can query historical data. All data processing and computing work is done by the PLC in this process [8]. The main workflow of PLC is shown in Fig. 4.

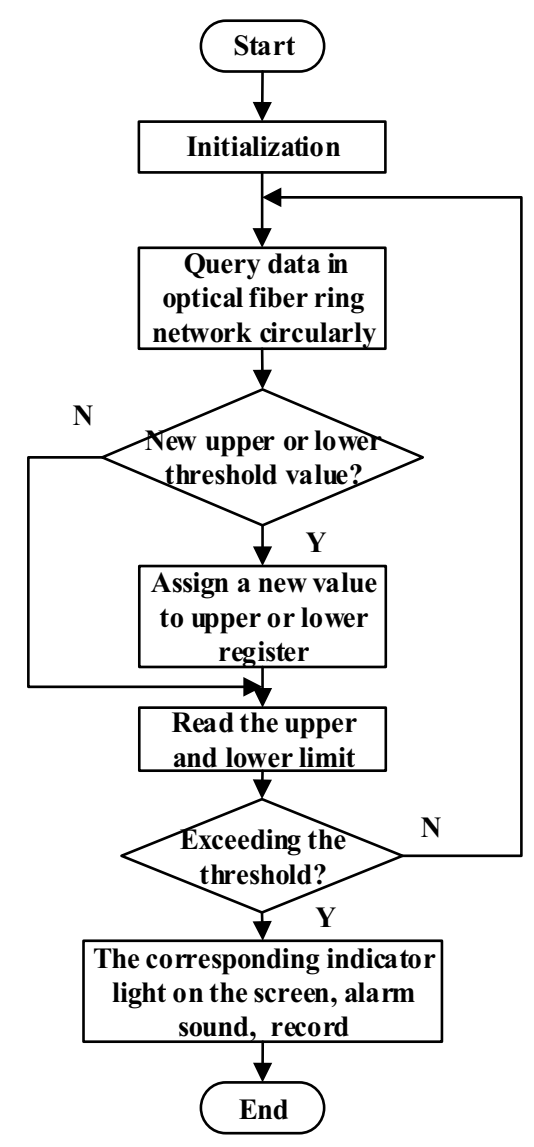

Figure 4. The main workflow of PLC.

\section{Experimental test and analysis}

In accordance with the system design scheme, the test samples we made are shown below (Fig. 5, Fig. 6 and Fig. 7). Fig. 5 shows the external and internal structure of the regional monitoring alarm while Fig. 6 shows the design of the regional monitoring panel and Fig. 7 shows the Human-machine interface of IPC.

In order to test the reliability of the system, we put the system in a large hospital which covers an area of $35000 \mathrm{~m} 2$. The hospital has four gas usage buildings and three medical gas supply stations. We deploy 100 regional monitoring alarms, 8 switches, and 7 sets of regional monitoring panel in the experiment, and the total fiber length is $2 \mathrm{~km}$. During several months' experiment, the system alarmed twice. We confirmed that the medical gas does go fault when alarm occurred, and monitoring center has an accurate positioning of fault node. Every part of the system works properly throughout the course of the experiment.
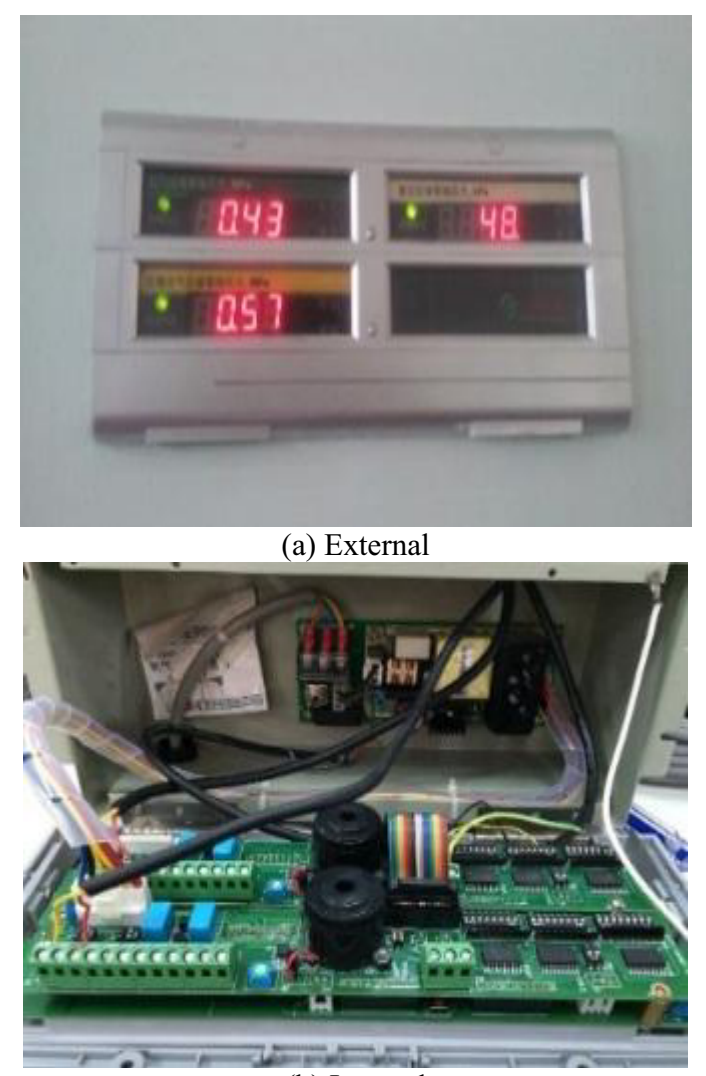

(b) Internal

Figure 5. Regional monitoring alarm

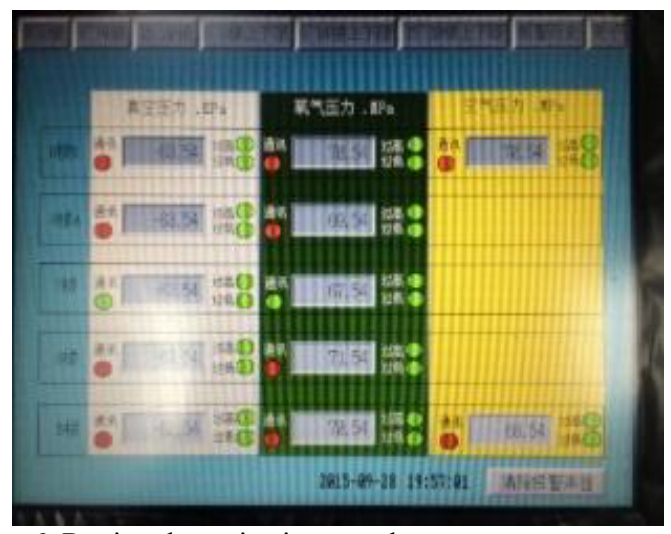

Figure 6. Regional monitoring panel.

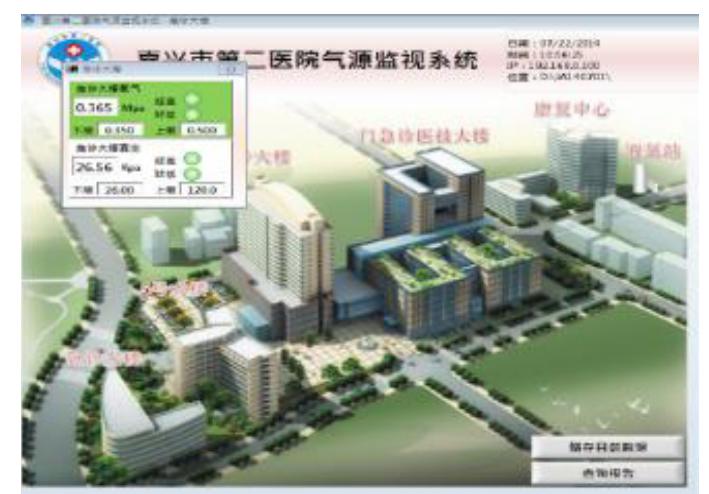

Figure 7. Human-machine interface of IPC

\section{Conclusions}


This paper implements a hospital medical gas monitoring system based on multi-mode fiber optic ring network, and elaborates on the content of system structure, hardware and software design and so on. Regional monitoring alarms are placed in all medical gas monitoring nodes, regional monitoring alarms connect each other with fieldbus. Regional monitoring panel is placed in every building to monitor the entire building. Multi-mode optical fiber ring network connects all regional monitoring buildings and medical gas supply stations, the IPC of monitoring center monitor all monitoring nodes of the entire hospital. Two means of communication fieldbus and multi-mode optical fiber ring network exist in one system, in order to make best use of the advantages and bypass the disadvantages so that the medical gas monitoring is more accurate and more stable. In conclusion, the system has a high social value and economic value. The future work will further extend our system both theoretically and experimentally.

\section{References}

1. S. L. Xu, L. B. Tan, J. Pu, Medical Gas Pressure Monitoring System Base on Modbus Communication Protocol, Ind. Control Comput. 23(8), 40-43 (2010)
2. N. J. Xu, Fault detection technique for wavelength division multiplexing passive optical network using chaotic fiber laser, Opt. Fiber Technol. 20, 163-167 (2014)

3. Z. Y. Liu, Design of communication protocol transform between Modbus RTU mode and TCP mode, Sci. Technol. Eng. 13(18), 1671-1815 (2013)

4. M. Grigoriu, M. C. Popescu, Study on Optical Fiber Insertion in Underground Telecommunication Networks Using Hydraulic Similarity, Procedia Technol. 22, 637-644 (2016)

5. S. C. Xu, S. Q. Yin, R. Srinivasan, M. Helander, Proactive Alarms Monitoring using Predictive Technologies, Comput. Aided Chem. Eng. 31, 15371541 (2012)

6. Q. S. He, AD conversion with high speed based on FPGA, Eletron. Des. Eng. 20(8), 122-124 (2012)

7. D. H. Chace, Medical Monitoring and Diagnosis, Encyclop. Mass Spectrom. 9, 305-311 (2016)

8. E. R. Alphonsus, M. O. Abdullah, A review on the applications of programmable logic controllers (PLCs), Renew. Sustain. Energy Rev. 60, 1185-1205 (2016) 